\title{
Array comparative genomic hybridization of 18 pancreatic ductal adenocarcinomas and their autologous metastases
}

\author{
Valentin Rausch ${ }^{1 *} \mathbb{D}$, Andreas Krieg ${ }^{1}$, Jordi Camps ${ }^{2,3}$, Bianca Behrens ${ }^{1}$, Manfred Beier ${ }^{4}$, Darawalee Wangsa ${ }^{2}$, \\ Kerstin Heselmeyer-Haddad ${ }^{2}$, Stephan E. Baldus ${ }^{5}$, Wolfram T. Knoefel ${ }^{1}$, Thomas Ried ${ }^{6+}$ \\ and Nikolas H. Stoecklein ${ }^{1+}$
}

\begin{abstract}
Background: Mortality rates of pancreatic cancer remain high, which is mainly due to advanced disease and metastasis. We hypothesized that genomic copy number alterations are enriched in metastatic cells compared to autologous primary tumors, which may inform on cancer-related pathways possibly serving as potential targets for specific therapies. We investigated 18 pancreatic ductal adenocarcinomas, including 39 lymph node and 5 distant metastases after surgical resection. Analysis was performed with array-based comparative genomic hybridization (aCGH).

Results: Metastases acquire a higher frequency of copy number alterations with the highest in distant metastasis ( median $=42$, lymph node metastases: median $=23$, primary tumors: median $=17$ ). In lymph node metastases, gains were prevalent on chromosome bands 8q11.23-q24.3, 12q14.1, 17p12.1, 21q22.12, and losses on 3p21.31, 4p14, 8p23.3-p11.21,17p12-11.2. Genes on amplified regions are involved in cancer-related pathways such as WNT-signaling, also involved in metastasis.
\end{abstract}

Conclusions: Pancreatic cancers show a high degree of intratumor heterogeneity, which could lead to resistance of chemotherapy and worse outcome. ACGH analysis reveals regions preferentially gained or lost in synchronous metastases encoding for genes involved in cancer-related pathways, which could lead to novel therapeutic opportunities.

Keywords: Intratumor hetereogeneity, Array-CGH, aCGH, Pancreatic cancer, PDAC, Pancreatic metastases

\section{Background}

Pancreatic cancer is characterized by a poor prognosis making it the fourth leading cause for cancer related death in the US today [1]. Every year, about 46,000 patients in the US develop pancreatic cancer with ductal adenocarcinoma (PDAC) as the most common histological type. The 5-year survival rate of this disease is still significantly below $10 \%$ [1], which could not be improved

\footnotetext{
*Correspondence: rauschv@gmail.com

'Thomas Ried and Nikolas H. Stoecklein contributed equally to this work

${ }^{1}$ Department of General, Visceral, and Pediatric Surgery, HeinrichHeine-University and University Hospital Duesseldorf, Moorenstrasse 5, 40225 Duesseldorf, Germany

Full list of author information is available at the end of the article
}

during the last decades despite novel therapeutic strategies [1]. Surgery still remains the only curative option, although 5-year-survival in potentially resectable patients does not exceed $20 \%$ [2]. This poor prognosis could make PDAC the second leading cancer death before 2030 [3]. One reason for this poor prognosis is the lack of early specific symptoms; many patients therefore present at stages in which metastases have already occurred, which are highly resistant to conventional chemotherapy [4].

The overall pattern of genetic alterations in pancreatic cancer is well studied: long-known are specific alterations in primary lesions that are acquired through a well-defined time course to invasive carcinomas and are maintained in cell lines $[5,6]$. Studies using whole 
genome sequencing have confirmed frequent mutations in prominent cancer associated genes, including $K R A S$, TP53, SMAD4 and CKDN2A [7-10]. In addition to these common targets, there exists a landscape of heterogeneous alterations of low frequency that may contain druggable targets [11]. Those studies showed a high degree of intratumor heterogeneity (ITH), which translates to a significant diversity in molecular mechanisms involved in tumor progression. However, ITH between samples from primary tumors and their corresponding metastases of the same patient has only been studied recently: Yachida [12] and Campbell [13], using sequencing techniques showed a high grade of ITH between metastases and the primary tumor obtained from the same patient. One important mechanism underlying the heterogeneity in PDAC is thought to be telomere dysfunction [14], resulting in breakage-fusion-bridge cycles, leading to amplification or deletion of specific regions. Hence, ITH is explained by the rearrangement of an instable genome. Moreover, centrosome amplifications were reported as a source of chromosomal instability and ITH [15]. The degree of chromosomal instability is also a clinical predictor of treatment response [16, 17].

In our study, we focused on differences in the genomic aberration profile of primary tumors and autologous and synchronous lymph node (LNM) or distant organ metastases $(\mathrm{OM})$. We hypothesized that cells that spread from primary PDAC and infiltrate lymph nodes or distant organs require a certain aberration profile. Since the presence of lymph node metastases is a poor prognostic factor in this disease [18], some of those features should also be present in loco-regional lymph node metastases. We expected that analysis of the genetic profile of primary tumors and their autologous lymph node metastases might reveal differences in those samples and therefore provide a panel of candidate genes that could be involved in promoting cancer cell metastasis.

In order to address these questions, we performed array-based comparative genomic hybridization (aCGH) to compare copy number alterations (CNAs) of primary tumors and their autologous metastases on a whole-genome level. This technique has already been used in other types of cancer, such as breast [1924] or colorectal cancer [25-27] to study differences between lymph node metastases and primary tumors. The identification of CNAs that specifically appear in metastasized tumor cells might not only lead to a better understanding of the genetic basis of PDAC metastasis but also unmask potential therapeutic targets that could provide a better efficacy against advanced disease, with the hope of improving the survival rates of PDAC patients.

\section{Methods}

\section{Clinical samples}

All patients who underwent surgery with curative intent for histologically confirmed PDAC between 2002 and 2009 at the Department for Surgery (A) at the University Hospital Duesseldorf were identified by medical chart review. The ethics committee of the Medical Faculty of the Heinrich-Heine-University Duesseldorf approved the investigation of FFPE material as performed in this investigation (vote Number 3821). Consent to participate was waived, since patient data were strictly pseudonymized and analysis had no detrimental effect on the participants. Inclusion criteria were: patients with microscopically confirmed metastases in more than one lymph node and PDAC at any localization within the pancreas, thus being classified at least as UICC stage IIb. All types of surgical resection were eligible. Exclusion criteria were as follows: other malignancies in the patient history, pancreatic tumors other than adenocarcinoma or adenocarcinoma of the Ampulla of Vater, neoadjuvant radio- or chemotherapy and very small lymph node metastases $(<0.3 \mathrm{~cm})$. Resectability of the tumor in surgery was not considered in the selection of the cases, thus cases with R1-resection were also included in the study. Applying these selection criteria, a total number of 52 patients were eligible to be included into our study.

The primary tumor was localized in the pancreatic head in 16 cases, one tumor extended from the head to the body of the pancreas and one tumor involved the pancreatic tail. The pancreatic tail tumor was surgically treated by resection of the distal pancreas, spleen and transverse colon including the left colonic flexure. Ten patients underwent the classical Whipple procedure (Pancreaticoduodenectomy) and seven patients underwent pylorus-preserving pancreaticoduodenectomy (PPPD). In all surgical procedures a lymph node dissection was performed resulting in a median number of 23 lymph nodes (range 12-55). Pathological examination of resected lymph nodes revealed a median number of 7 LNM (range 3-41). Tumor-free resection margins were achieved in 11 patients. After quality control, 18 patients were randomly selected from whom formalin-fixed and paraffin-embedded (FFPE) tissue specimen of the respective primary tumors (PT) and a total number of 39 lymph node metastases (LNM) were available for further analysis. The median age of our cohort was 64 years (range 44-81 years) (Table 1). In 4 cases, a total number of 5 liver metastases were found intraoperatively. In these cases, metastases had not been discovered preoperatively. Distant metastases were defined as organ metastases $(\mathrm{OM})$ other than the pancreas and were also included in our analysis. 
Table 1 Clinical and pathological data of patients included in study

\begin{tabular}{|c|c|c|c|c|c|c|c|c|c|c|c|c|}
\hline Patient (no.) & Age at procedure & LNM $^{a}$ (no.) & $\mathrm{LN}^{\mathrm{b}}$ total (no.) & $\mathrm{OM}^{\mathrm{c}}$ & $T$ & $\mathrm{~N}$ & $M$ & Grad. & $\mathbf{R}$ & Stage & Localization & Procedure \\
\hline 2 & 54 & 10 & 18 & 1 & 4 & 1 & 1 & 2 & 0 & IV & Head & Whipple \\
\hline 8 & 62 & 30 & 37 & 1 & 3 & 1 & 1 & 3 & 0 & IV & Head & Whipple \\
\hline 19 & 76 & 7 & 35 & 0 & 3 & 1 & 0 & 3 & 0 & $\| \mathrm{b}$ & Head & Whipple \\
\hline 24 & 44 & 7 & 19 & 1 & 3 & 1 & 1 & 3 & 1 & IV & Head & PPPD \\
\hline 28 & 65 & 3 & 39 & 1 & 3 & 1 & 1 & 3 & 0 & IV & Head & PPPD \\
\hline 33 & 67 & 6 & 27 & 1 & 3 & 1 & 1 & 3 & 1 & IV & Head & PPPD \\
\hline 41 & 66 & 7 & 22 & 0 & 3 & 1 & 0 & 2 & 0 & $1 \mathrm{lb}$ & Head & Whipple \\
\hline 42 & 75 & 3 & 23 & 0 & 3 & 1 & 0 & 3 & 0 & IIb & Head, body & Whipple \\
\hline 44 & 55 & 8 & 16 & 0 & 3 & 1 & 0 & 3 & 0 & Ilb & Head & PPPD \\
\hline 45 & 62 & 41 & 55 & 1 & 3 & 1 & 1 & 2 & 1 & IV & Head & Whipple \\
\hline 47 & 81 & 7 & 12 & 0 & 3 & 1 & 0 & 3 & 1 & $\| \mathrm{b}$ & Head & Whipple \\
\hline 48 & 64 & 9 & 32 & 0 & 4 & 1 & 0 & 3 & 1 & III & Head & PPPD \\
\hline 49 & 79 & 17 & 50 & 1 & 3 & 1 & 1 & 3 & 1 & IV & Head & Whipple \\
\hline 50 & 47 & 4 & 22 & 1 & 3 & 1 & 1 & 3 & 1 & IV & Head & PPPD \\
\hline 51 & 59 & 4 & 24 & 1 & 3 & 1 & 0 & 3 & 0 & IV & Head & PPPD \\
\hline 52 & 61 & 8 & 12 & 0 & 3 & 1 & 0 & 3 & 0 & Ilb & Head & Whipple \\
\hline 53 & 61 & 3 & 36 & 1 & 3 & 1 & 1 & 2 & 0 & IV & Tail & Distal pancreatectomy \\
\hline 54 & 72 & 5 & 23 & 0 & 3 & 1 & 0 & 3 & 0 & Ilb & Head & Whipple \\
\hline
\end{tabular}

a Lymph node metastases

b Lymph nodes

c Distant metastases

\section{DNA extraction}

Genomic DNA was isolated from FFPE tissue specimens of primary tumors, LNMs and OMs as recently described [52]. Therefore, hematoxylin and eosin (HE) stained sections of all specimens were reviewed by a pathologist (Baldus, SE) who marked representative tumor regions on the microscope slide (Fig. 1). Serial $10 \mu \mathrm{m}$ thick sections were then processed from the respective FFPE blocks. Marked microscope slides served to localize the tumor region on the respective
FFPE block. DNA extraction was performed using the Qiagen QIAmp DNA FFPE Tissue Kit (Qiagen, Hilden, Germany) according to manufacturer's instructions. As reference DNA for aCGH analysis we extracted tissue from histologically confirmed normal lymph nodes of the corresponding patient to avoid copy number variations of non-pathological regions to interfere with our results. Purity and concentration of the DNA was measured spectrophotometrically and integrity tested on a $1 \%$ agarose gel.

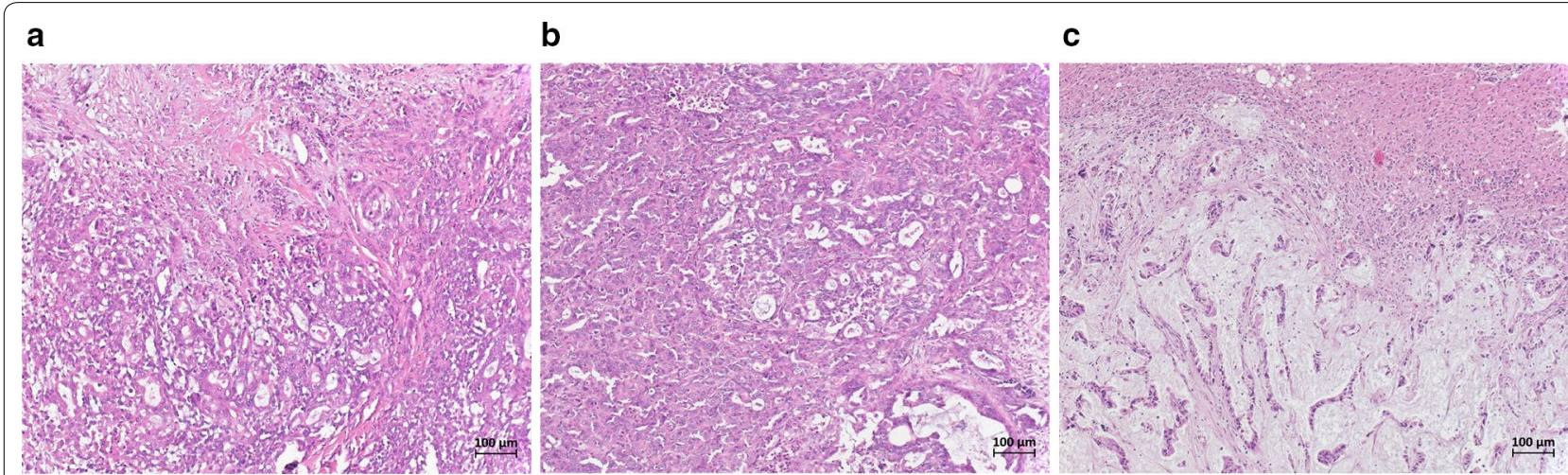

Fig. 1 H\&E stained samples from PDAC. a Sample section from a primary tumor (case 33). b Sample section from one of the lymph node metastasis (case 33). c Sample section from a distant metastasis (lung) (case 33) 


\section{Array comparative genomic hybridization}

Before array CGH was performed, DNA was RNase (RNase, DNase free, Roche, USA) treated and purified a second time before labeling using Kreatech's (Kreatech Diagnostics, Amsterdam, Netherlands) non-enzymatic Universal Linkage System (ULS) according to the manufacturers' instructions. We used reversed labeling for our samples: ULS-Cy3 to label sample-DNA and ULS-Cy5 to label the reference DNA. After purification of the labeled DNA and quality control with spectrophotometry, the DNA was analyzed with aCGH using $8 \times 60 \mathrm{~K}$ arrays from Agilent's SurePrint G3 Human CGH Microarray Kit (Agilent Technologies, Santa Clara, CA, USA). Hybridized slides were then washed and scanned by the Agilent SureScan Microarray Scanner System. Normalized, quality filtered $\log 10$-ratios were obtained using Agilent Feature Extraction software. Initial data visualization was performed using Agilent Genomic Workbench (version 6.5.0.18).

\section{Data analysis}

Further bioinformatic analysis was accomplished using an R-pipeline [53] composed by several $\mathrm{R}$ add-ons available from Bioconductor (bioconductor.org) [54] and CRAN (cran.r-project.org) projects. Prior to this analysis, the $\log 10$ ratios from the Agilent Feature Extraction software were converted to $\log 2$ ratios. Improper oligonucleotides were removed in quality control using the same flags as in the Agilent Feature Extraction tool: oligonucleotides were removed if gIsSaturated $=1$ or rIsSaturated $=1$ to exclude over- or undersaturated spots, gIsFeatNonUnifOL $=1$ or rIsFeatNonUnifOL $=1$ or $\log$ Ratio $=0$ to exclude spots that exhibited implausible information, meaning intensities of individual pixels on one spot were not homogeneous enough or log ratio was set to 0 if the background exceeded the signal intensity.

For breakpoint detection we used the "lawsglad"-algorithm [55] from the GLAD-function [56]. We applied the default parameters, except median center $=$ FALSE, since centering of the arrays is carried out before analyzing the data in our pipeline. Only calls of aberrant regions spanning at least three oligos and with a minimum absolute $\log 2$-ratio of 0.2 were considered for further analysis. Hence, increases in sample to reference-ratio $>1.2$ were defined as gains, decreases in sample to reference ratio $<0.8$ were defined as losses. Since our main focus was the general differentiation of altered regions between tumor cells from different sites, no further distinction was made for high-level amplification or homozygous losses. Overviews of the genome were generated from this data with a modified plot-function of the aCGHdatabase. Shared aberrations were defined as any region in two or more samples that was deleted or amplified.
Private aberrations were defined as those only present in one sample. For visualization of the data we used a modified plot function of the "aCGH"-package from Bioconductor project.

Statistical differences in the number of CNA were assessed using nonparametric testing (Mann-Whitney $U$ test). A p $<0.05$ was defined as statistical significance.

Enriched genetic alterations in metastatic cells were found by comparing the appearance of each CNA in primary tumors and corresponding lymph node metastases or distant metastases. Enrichment was defined as significant differences in frequencies of CNA in aCGH-data of two corresponding samples. Significance was assessed with a standard Fishers exact test for $3 \times 2$ contingency tables (gain, loss, no change in two samples). Significantly enriched $(p \leq 0.05)$ alterations present in $>25 \%$ of respective the samples were then analyzed with Panther classification system (www.pantherdb.org) [57].

For calculation of the genetic distance of the samples, aberrations were classified as shared (present in more than one sample) or private (distinct alterations in one sample). Asymmetric binary distance between the samples was then calculated and plotted in a matrix. The binary distance was calculated by dividing all private regions in two arrays through all segmented regions; this is represented as a non-dimensional number between 0.0 and 1.0. Hence, higher values indicate a larger binary distance and therefore a higher level of heterogeneity between two distinct samples. The matrix of the binary distance data was used to calculate the mean distance between two samples of several subsets of samples. Differences in mean distances were tested for statistical significance using a Mann-Whitney-U test.

\section{Results}

CNAs of primary tumors and metastases

Profiles from the aCGH-data displayed copy number alterations (CNAs) in all primary tumors and metastases (Fig. 2a, b, see also Additional file 1: Figure S1A-C). However, one sample (case45_PT1) exhibited alterations with less than three oligonucleotides and was therefore excluded from further analysis.

CNAs were defined as gains (more than 1.2 in sample to reference ratio) or losses (less than 0.8 in sample to reference ratio). Although not statistically significant, distant organ metastases $(\mathrm{OM}, \mathrm{n}=5)$ displayed the highest number of CNAs (median CNAs: 42, 1-124), followed by lymph node metastases (LNM, $\mathrm{n}=39$; median CNAs: 23, 1-104) and primary tumors (PT, $\mathrm{n}=18$; median CNAs: $17,0-66)$. In addition, we observed a tendency toward a higher frequency of gains in the primary tumors with a median of 13 gained regions (0-95) compared to a median of 9.5 losses $(0-42)(\mathrm{p}=0.0709)$. 


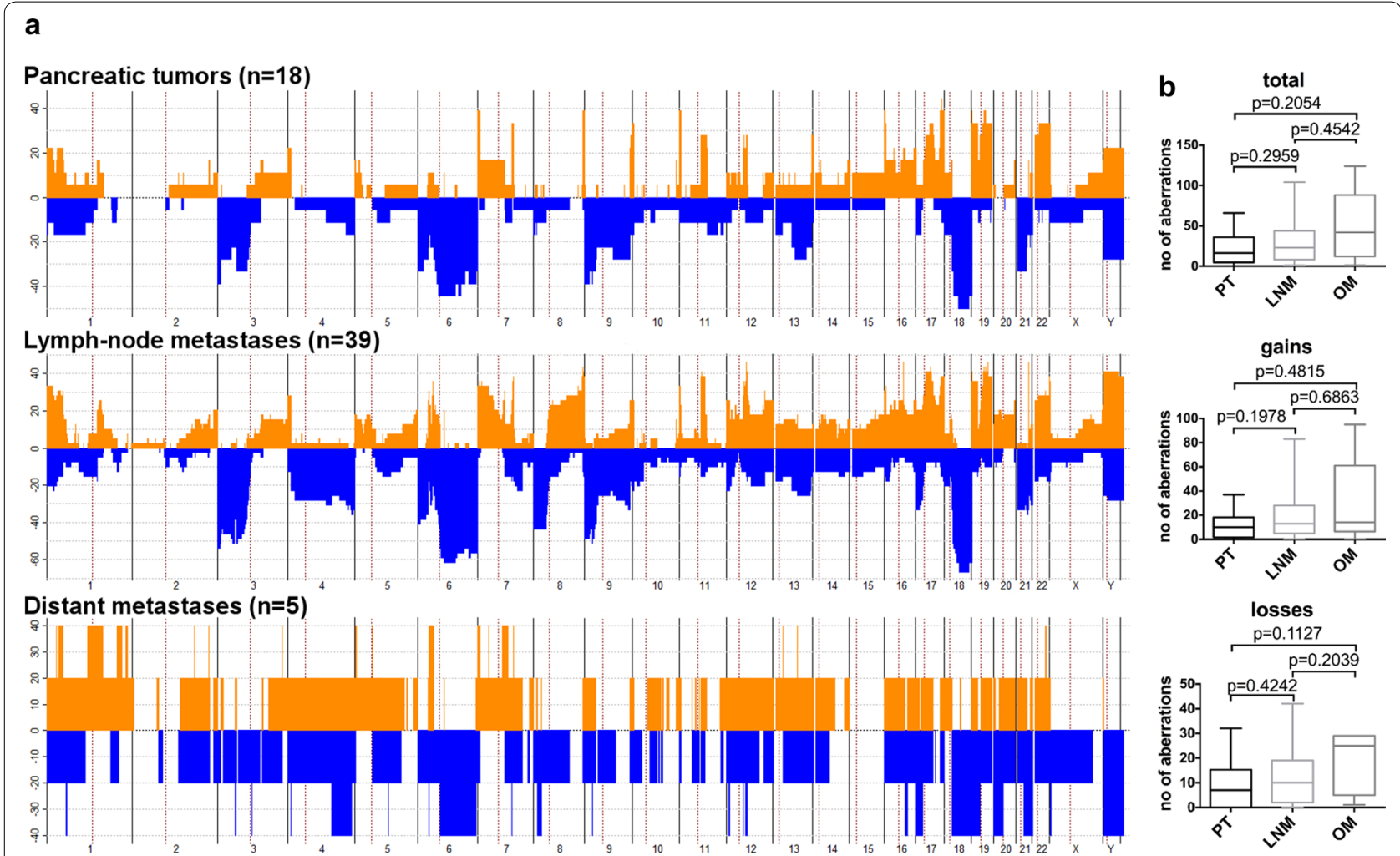

Fig. 2 a Penetrance plot of genomic alterations depending on localization. b Total number of median gains and losses in primary tumors (PT), lymph node metastases (LNM) and distant metastases (OM)

The regions most commonly gained in primary tumors mapped to chromosome bands $17 \mathrm{q} 25.1$ (44.4\%), $7 \mathrm{p} 22.3$ (38.9\%), 8q24.3 (38.9\%), 11p15.5 (38.9\%), 16p13.3 (38.9\%), 17q24.3-q25.3 (38.9\%), 19p13.3 (38.9\%), 19q13.12-q13.2 (38.9\%). The most commonly lost regions were 18q12.3q22.3 (50\%), 6p11.2-q13 (44.4\%), 6q13-q22.31 (44.4\%), 18q12.1-q12.3 (44.4\%), 18q22.3-q23 (44.4\%).

Next, we compared differences in CNAs between primary tumors, LNM and OM that were enriched in more than $25 \%$ of the samples (Additional file 2: Table S1A-H), since enrichment of specific regions could point to selected cancer-related genes. When comparing enriched gains between primary tumors and lymph node metastases, specific gains for lymph node metastases became evident on chromosome bands 8q11.23-q24.3, $12 \mathrm{q} 14.1,17 \mathrm{p} 12.1$ and $21 \mathrm{q} 22.12$. In addition, losses that could be observed only in lymph node metastases were on 3p21.31, 4p14, 8p23.3-p11.21 and 17p12-11.2 (Fig. 3). In contrast, distant metastases exhibited enriched gains on 1q32.1-q42.2 and 5q11.1, as well as enriched losses at $4 \mathrm{q} 28.1-\mathrm{q} 32.3,16 \mathrm{q} 21,17 \mathrm{p} 11.2$ and $20 \mathrm{p} 13-\mathrm{q} 11.21$ that could not be detected in matched primary tumors. Whereas enriched losses on chromosome 3p26.1-25.3 and 18q21.1-23 were specific for LNM when compared with OM, losses on chromosome 1p13.1-12 and 1q32.1q41 and gains on chromosome 20p11.1-p11.21 were specifically present in OM. To get insight whether enriched genes in LNMs compared to PTs were involved in known cancer-related pathways, we performed gene ontology analysis on enriched regions using the Panther server (Additional file 2: Table S2). In total, 38 pathways were affected in enriched gains of LNM, 42 pathways were affected in enriched losses of LNM. Pathways most commonly affected with more than 2 genes in enriched losses of LNM were: FGF signaling, Apoptosis signaling, Angiogenesis, p53 signaling, Wnt signaling, and Gonadotropin releasing hormone receptor signaling. Only the Wntsignaling pathway was affected with more than 2 genes in enriched gains of LNM.

\section{Comparison between CNAs of primary tumors and their matched metastases}

Since we noted differences in the number of aberrations and the aberration profiles between primary tumors and their metastases, we investigated samples for their shared genetic aberrations in order to better describe heterogeneity of the samples. To quantify heterogeneity, we measured the mean binary distance between different subsets 


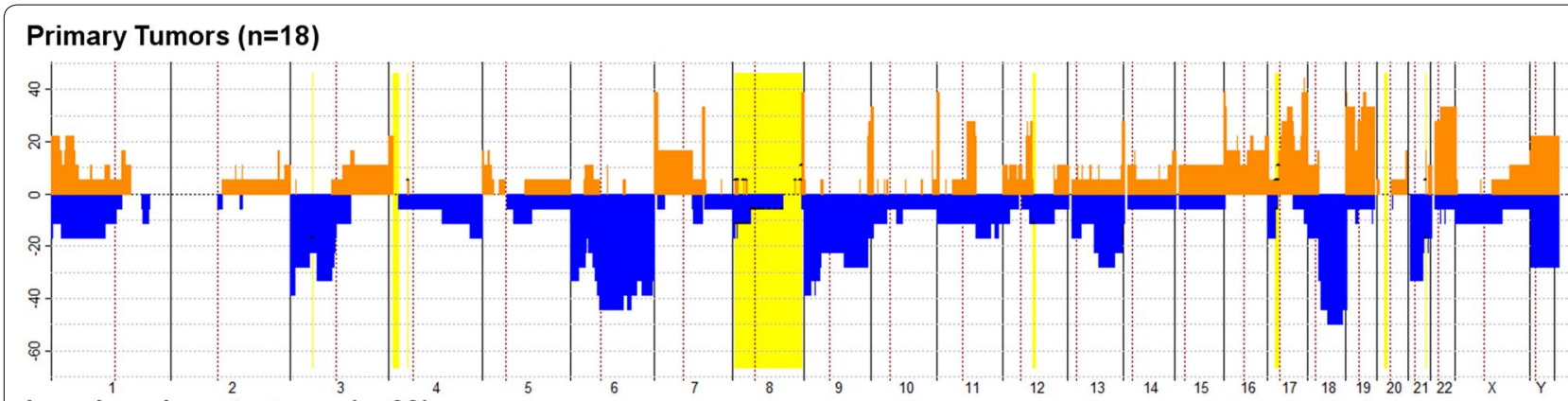

Lymph-node metastases $(n=39)$

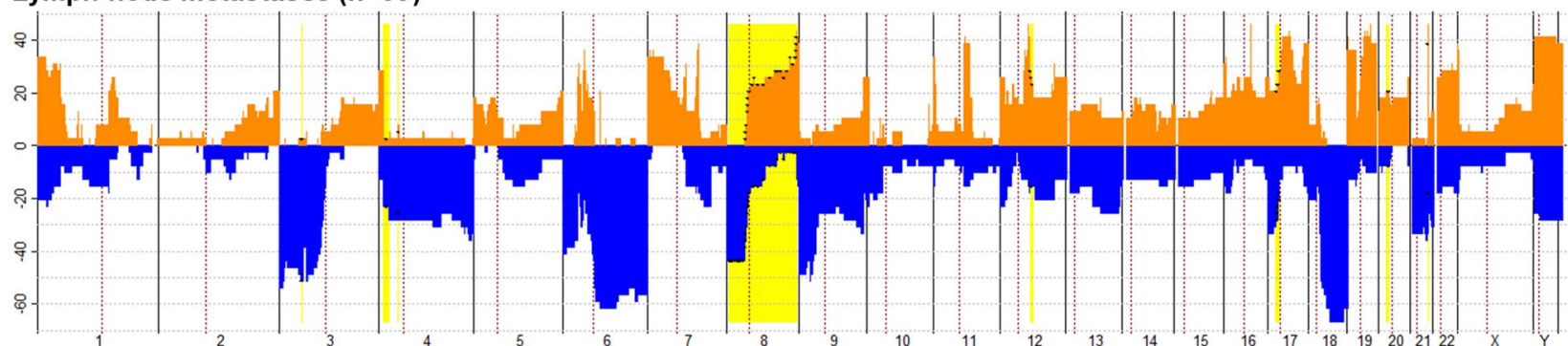

Fig. 3 Penetrance plots of primary tumors (PT), and lymph node metastases (LNM). Yellow lines show significant enriched alterations in LNM

(PT vs. corresponding LNM, between LNM throughout cases) as described above: the mean binary distance (on a scale ranging from 0 to 1 , whereas $1=$ no shared alterations, $0=$ all alterations shared) between primary tumors and their corresponding lymph node metastases (Fig. 4) showed a significant higher $(p=0.0128)$ degree of heterogeneity $(0.74$, range $0.10-1.0)$ than between different lymph node metastases throughout all cases $(0.87$, range $0.07-1.0)$. Mean heterogeneity between LNM and their corresponding OM was also high (binary distance 0.77 , range $0.32-1$ ).

In individual cases we could find both, examples for highly heterogeneous and only slightly heterogeneous

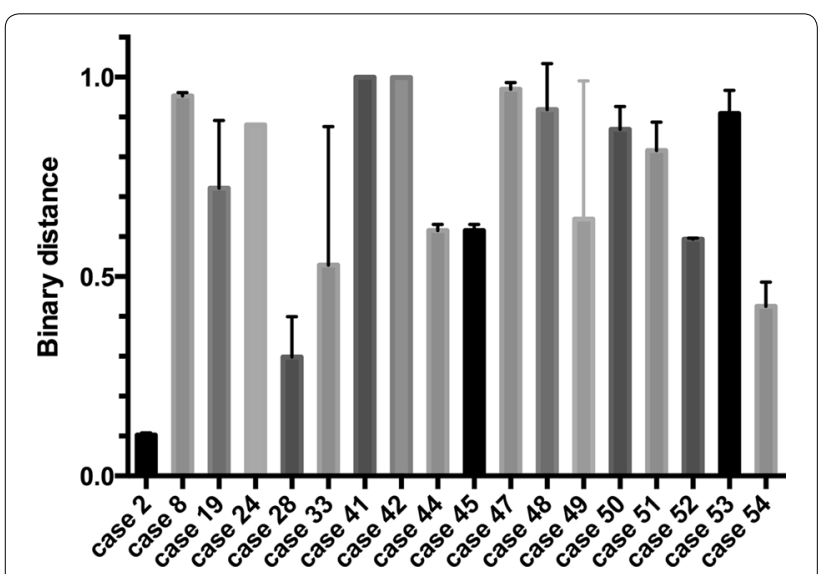

Fig. 4 Mean binary distance and range of each primary tumor to their corresponding lymph node metastases tumors: e.g. binary distance could be shown to spread widely in case 49, distance between LNM and corresponding $\mathrm{OM}$ ranged between 0.96 and 1.0, indicating a large heterogeneity between all samples from this case, whereas distance in case 24 ranged between 0.32 and 0.35 , indicating only a small grade of heterogeneity.

\section{Discussion}

Here we compared copy number gains and losses of primary PDAC tumors with their matched LNM and OM using aCGH. The overall CNA pattern of all samples was typical for PDAC [28-31]. However, while the aCGH data clearly showed that genetic heterogeneity between individual cases was higher than between different samples of an individual case, the CNA patterns of metastases diverged to some extent from their matched primary tumors. ITH, as indicated by heterogeneity between metastases and primary tumors from the same case is high in our cases, with a mean heterogeneity index of 0.74 . Among metastases, gains on chromosome bands 8q24, 12q13, 16q12, 19q13, 21q22, 17q21 and losses at $18 \mathrm{q}$ and $6 \mathrm{q} 13$ were the most common aberrations. Most frequently enriched was gain of chromosome $8 \mathrm{q}$ $(\mathrm{p}<0.043)$, comprising important cancer related genes such as MYC or FZD6. These genes are associated with an upregulation of Wnt-signaling, which is known to be altered in PDAC [11]. In addition to its role as potent oncogenic driver, MYC [32-36] contributes to chromosomal instability [37], which again can drive ITH. Gain of chromosome 8q has moreover been described in other 
cancers: in oral squamous cell cancer gain of 8q could be found to be associated with lymph-node metastasis [38], in colorectal cancer this alteration occurred significantly more frequent in corresponding brain metastases [39] and in breast cancer gain of MYC defines the transition from pre-invasive ductal carcinoma to invasive ductal carcinoma [40]. In view of these data, the involved gained 8q genes might be interesting candidates for metastatic drivers in PDAC. Gene ontology analysis of involved pathways hinted that several pathways could possibly be affected by gained or lost regions in lymph node metastases compared to primary tumors. Genes such as MYC play an important role in pathways relevant for cancer development such as cell-cycle control through Wntsignaling. However, gene ontology analysis does not allow to differentiate between 'driver-'and 'passenger'mutations and could therefore only be used to screen for candidates that could be studied in future studies.

Clearly, technical issues of the aCGH-methodology could also contribute to our observed differences. Despite our use of manual micro-dissection, the overall smaller number of alterations in primary tumors could result from the limitations of using bulk DNA for aCGH, leading to an average profile of the genetic alterations from several subclones. Moreover, only small regions of the primary tumor were extracted for aCGH analysis, hence not the entirety of clones is reflected with this method. Also, especially in PDAC with its strong desmoplastic stromal response, the amount of analyzed non-malignant cells can be significant.

Genetic heterogeneity between primary PDAC and their distant metastases as shown in our study could, however, also be observed on the sequence level using next generation sequencing (NGS): Yachida et al. [12] analyzed somatic mutations after extracting samples from different metastatic locations in seven PDAC patients and could detect the majority (mean 64\%) of mutations in all samples from a patient. However, in two patients they collected samples from multiple sites of the sectioned primary tumor and found that subclones present in the primary tumor already reflected heterogeneity that was observed in the metastases, suggesting tumor spread to occur late in the course of the disease. In contrast, the study by Campbell et al. [13] who also sequenced samples from primary tumors and metastases of pancreatic cancer observed alterations, including driver mutations, that were exclusive in metastases, suggesting an earlier time point of dissemination followed by parallel clonal evolution of metastatic clones. These findings were possibly corroborated by data from experiments with a transgenic PDAC mouse model demonstrating that dissemination of cancer cells can be an early event [41], even occurring at the stage of pre-neoplastic lesions. In our study, we observed cases in which metastases were genetically close to their primary tumors but also more distant examples. Most probably, both scenarios can occur in PDAC patients explaining the observed different CNA patterns between primary tumors and their metastases: (a) cancer cells that leave the primary tumor (early) undergo additional changes due to clonal selection and adaptation at the metastatic site and (b) small subpopulations evolved within the primary tumor with the propensity to metastasize become the dominant metastatic clone. However, similarities between tumor samples from the same case might also be explained to some extent by other mechanisms, such as self-seeding. The hypothesis of self-seeding describes a mechanism in which metastases, once they infiltrated a distant organ, could seed themselves and re-enter their primary tumor [42].

Interestingly, ITH as a result of adaption processes can also be observed when sampling the same tumor over time [43-47]. Such genetic ITH enables more effective adaption to new microenvironments [48], e.g., at distant sites, and facilitates the development of therapeutic resistance $[43,49,50]$. In this context it is notable that increased levels of ITH are correlated to higher aggressiveness reflected by shorter survival in some cancer types [51]. It would be therefore highly interesting for future studies, to investigate, whether ITH in PDAC is a biomarker for aggressiveness and therapeutic resistance.

\section{Conclusions}

Our comparative study of matched primary and metastatic PDAC tissue showed different levels of ITH and revealed CNAs that were enriched in metastatic lesions. These organ specific alterations might facilitate the identification of metastatic drivers in subsequent future studies, which might be amenable for future therapeutic interventions.

\section{Additional files}

Additional file 1: Figure S1. ACGH-Profiles of each sample. The log-2-ratio is displayed in the $y$-axis, the localization on the genome is displayed on the $x$-axis. Called gains are marked orange, called losses are marked blue.

Additional file 2: Table S1. Enriched Gains and Losses (percentage of altered samples, range and involved Genes). A: Enriched Gains in LNM vs. PT. B: Enriched Losses in LNM vs. PT. C: Enriched Gains in OM vs. PT. D: Enriched Losses in PT vs. OM. E: Enriched Losses in LNM vs. OM. F: Enriched Gains in OM vs. LNM. G: Enriched Losses in OM vs. LNM. Table S2. Involved pathways, genes and respective protein class in enriched alterations. A: Altered pathways in enriched Gains (LNM vs PT). B: Altered pathways in enriched Losses (LNM vs PT). 


\begin{abstract}
Abbreviations
aCGH: array-comparative genomic hybridization; CNA: copy number alteration; FFPE: formalin-fixated, paraffin-embedded; ITH: intratumor heterogeneity; LNM: lymph-node metastasis; NGS: next-generation sequencing; OM: distant metastasis; PDAC: pancreatic ductal adenocarcinoma; PPPD: pyloruspreserving pancreaticoduodenectomy; PT: primary tumor; UICC: Union internationale contre le cancer.
\end{abstract}

\section{Authors' contributions}

VR carried out the case-selection, histological sighting, DNA extraction, genetic experiments, statistical analysis and wrote the manuscript. AK participated in the design of the study and in writing the manuscript. JC participated in the design and analysis of the genetic studies. BB participated in the DNA extraction and supported with the statistical analysis. MB participated in the statistical analysis of the aCGH-data. DW participated in the genetic experiments and rendered technical support. $\mathrm{KHH}$ advised statistical analysis of the genetic experiments and rendered technical support for the experiments. SEB participated in the histological confirmation of the pancreatic cancer. WTK conceived of the study and participated in the manuscript. TR and NHS both conceived the study, participated in the design helped with the manuscript and statistical analysis. All authors read and approved the final manuscript.

\section{Author details}

1 Department of General, Visceral, and Pediatric Surgery, Heinrich-Heine-University and University Hospital Duesseldorf, Moorenstrasse 5, 40225 Duesseldorf, Germany. ${ }^{2}$ Genetics Branch, Center for Cancer Research, National Cancer Institute, National Institutes of Health, Bethesda, MD, USA. ${ }^{3}$ Present Address: Gastrointestinal and Pancreatic Oncology Group, Institut d'Investigacions Biomèdiques August Pi i Sunyer (IDIBAPS), Centro de Investigación Biomédica en Red de Enfermedades Hepáticas y Digestivas (CIBEREHD), Hospital Clínic de Barcelona, Barcelona, Spain. ${ }^{4}$ Institute of Human Genetics and Anthropology, Heinrich-Heine-University and University Hospital, Duesseldorf, Germany. ${ }^{5}$ Department of Pathology, Heinrich-Heine-University and University Hospital, Duesseldorf, Germany. ${ }^{6}$ Section of Cancer Genomics, Genetics Branch, Department of Health and Human Services, National Cancer Institute, National Institutes of Health, Center for Cancer Research, Bethesda, MD, USA.

\section{Acknowledgements}

Amanda Bradley provided technical support on experimental background research. Oda C. Goetzke provided editorial support in the development of this manuscript.

\section{Competing interests}

The authors declare that they have no competing interests.

\section{Availability of data and materials}

The original aCGH-data used and analysed during the current study are available from the corresponding author on reasonable request. They are publically available at the NCBI Gene Expression Omnibus (GEO) Accession Number GSE103787. http://www.ncbi.nlm.nih.gov/geo/query/acc.cgi?acc=GSE103787.

\section{Consent for publication}

Not applicable.

\section{Ethics approval and consent to participate}

This retrospective work was done in accordance with a protocol for archival tissue collection and use which was approved by the ethics committee of the Medical Faculty of the Heinrich-Heine-University Duesseldorf. The requirement for a patient consent form was waived by the ethics committee of the Medical Faculty of the Heinrich-Heine-University Duesseldorf (Vote Number 3821).

\section{Funding}

For this study, BB, WTK, MB, SB and NHS received funding as staff of the University Hospital Duesseldorf. DW, $\mathrm{KHH}$ and TR received funding as staff of the National Institutes of Health, National Cancer Institute. JC, at the time of the conduction of the experiments, also received funding as staff of the National Institutes of Health, National Cancer Institute and obtained a grant from Instituto de Salud Carlos III co-funded by the European Regional Development Fund (ERDF) (CP13/00160), and the European Commission (COLONGEVA). No specific funding for this study has been granted for all of these authors. VR has received a travel-grant from the Hedwig and Waldemar Hort scholarship foundation. This work was supported in parts by the Intramural of the National Institutes of Health, National Cancer Institute, Center for Cancer Research, Bethesda, MD.

\section{Publisher's Note}

Springer Nature remains neutral with regard to jurisdictional claims in published maps and institutional affiliations.

Received: 25 September 2016 Accepted: 31 October 2017

Published online: 06 November 2017

\section{References}

1. Siegel RL, Miller KD, Jemal A. Cancer statistics, 2015. CA Cancer J Clin. 2015:65:5-29.

2. Warshaw AL, Fernandez-del Castillo C. Pancreatic carcinoma. N Engl J Med. 1992;326:455-65.

3. Rahib L, Smith B, Aizenberg R. Projecting cancer incidence and deaths to 2030: the unexpected burden of thyroid, liver, and pancreas cancers in the United States. Cancer Res. 2014; 74: 2913.

4. Haeno H, Gonen M, Davis MB, Herman JM, lacobuzio-Donahue CA, Michor F. Computational modeling of pancreatic cancer reveals kinetics of metastasis suggesting optimum treatment strategies. Cell. 2012;148:362-75.

5. Aguirre AJ, Brennan C, Bailey G, Sinha R, Feng B, Leo C, et al. High-resolution characterization of the pancreatic adenocarcinoma genome. Proc. Natl. Acad. Sci. U. S. A. 2004/06/17. 2004;101:9067-72.

6. Hruban RH, Goggins M, Parsons J, Kern SE. Progression model for pancreatic cancer. Clin Cancer Res. 2000;6:2969-72.

7. Maitra A, Hruban RH. Pancreatic cancer. Annu Rev Pathol. 2008;3:157-88.

8. Mimeault M, Brand RE, Sasson AA, Batra SK. Recent advances on the molecular mechanisms involved in pancreatic cancer progression and therapies. Pancreas. 2005;31:301-16.

9. Biankin AV, Waddell N, Kassahn KS, Gingras MC, Muthuswamy LB, Johns $\mathrm{AL}$, et al. Pancreatic cancer genomes reveal aberrations in axon guidance pathway genes. Nature. 2012;491:399-405.

10. Jones S, Zhang X, Parsons DW, Lin JC, Leary RJ, Angenendt P, et al. Core signaling pathways in human pancreatic cancers revealed by global genomic analyses. Science. 2008;321:1801-6.

11. Waddell N, Pajic M, Patch AM, Chang DK, Kassahn KS, Bailey P, et al. Whole genomes redefine the mutational landscape of pancreatic cancer. Nature. 2015;518:495-501.

12. Yachida S, Jones S, Bozic I, Antal T, Leary R, Fu B, et al. Distant metastasis occurs late during the genetic evolution of pancreatic cancer. Nature. 2010;467:1114-7.

13. Campbell PJ, Yachida S, Mudie L, Stephens PJ, Pleasance ED, Stebbings $L A$, et al. The patterns and dynamics of genomic instability in metastatic pancreatic cancer. Nature. 2010;467:1109-13.

14. O'Hagan R, Chang S, Maser R. Telomere dysfunction provokes regional amplification and deletion in cancer genomes. Cancer Cell. 2002;2:149-55.

15. Sato N, Mizumoto K, Nakamura M, Maehara N, Minamishima YA, Nishio S, et al. Correlation between centrosome abnormalities and chromosomal instability in human pancreatic cancer cells. Cancer Genet Cytogenet. 2001;126:13-9.

16. Holohan C, Van Schaeybroeck S, Longley DB, Johnston PG. Cancer drug resistance: an evolving paradigm. Nat Rev Cancer. 2013;13:714-26.

17. Lee AJX, Endesfelder D, Rowan AJ, Walther A, Birkbak NJ, Futreal PA, et al. Chromosomal instability confers intrinsic multidrug resistance. Cancer Res. 2011;71:1858-70

18. Kanda M, Fujii T, Nagai S, Kodera Y, Kanzaki A, Sahin TT, et al. Pattern of lymph node metastasis spread in pancreatic cancer. Pancreas. 2011:40:951-5.

19. Vollebergh MA, Klijn C, Schouten PC, Wesseling J, Israeli D, Ylstra B, et al. Lack of genomic heterogeneity at high-resolution aCGH between primary breast cancers and their paired lymph node metastases. PLoS ONE. 2014;9:e103177. 
20. Kuukasjarvi T, Karhu R, Tanner M, Kahkonen M, Schaffer A, Nupponen N, et al. Genetic heterogeneity and clonal evolution underlying development of asynchronous metastasis in human breast cancer. Cancer Res. 1997;57:1597-604.

21. Desouki MM, Liao S, Huang H, Conroy J, Nowak NJ, Shepherd L, et al. Identification of metastasis-associated breast cancer genes using a highresolution whole genome profiling approach. J Cancer Res Clin Oncol. 2011;137:795-809.

22. Friedrich K, Weber T, Scheithauer J, Meyer W, Haroske G, Kunze KD, et al. Chromosomal genotype in breast cancer progression: comparison of primary and secondary manifestations. Cell Oncol. 2008;30:39-50.

23. Nishizaki T, DeVries S, Chew K, Goodson WH, Ljung BM, Thor A, et al. Genetic alterations in primary breast cancers and their metastases: direct comparison using modified comparative genomic hybridization. Genes Chromosom Cancer. 1997;19:267-72.

24. Wang C, lakovlev VV, Wong V, Leung S, Warren K, lakovleva G, et al. Genomic alterations in primary breast cancers compared with their sentinel and more distal lymph node metastases: an aCGH study. Genes Chromosom Cancer. 2009:48:1091-101.

25. Aragane H, Sakakura C, Nakanishi M, Yasuoka R, Fujita Y, Taniguchi H, et al. Chromosomal aberrations in colorectal cancers and liver metastases analyzed by comparative genomic hybridization. Int J Cancer. 2001;94:623-9.

26. Al-Mulla F, Keith WN, Pickford IR, Going JJ, Birnie GD. Comparative genomic hybridization analysis of primary colorectal carcinomas and their synchronous metastases. Genes Chromosom Cancer. 1999;24:306-14.

27. Roessler S, Lin G, Forgues M, Budhu A, Hoover S, Simpson RM, et al. Integrative genomic and transcriptomic characterization of matched primary and metastatic liver and colorectal carcinoma. Int J Biol Sci. 2015;11:88-98.

28. Aguirre AJ, Brennan C, Bailey G, Sinha R, Feng B, Leo C, et al. High-resolution characterization of the pancreatic adenocarcinoma genome. Proc Natl Acad Sci USA. 2004;101:9067-72.

29. Harada T, Baril P, Gangeswaran R, Kelly G, Chelala C, Bhakta V, et al. Identification of genetic alterations in pancreatic cancer by the combined use of tissue microdissection and array-based comparative genomic hybridisation. Br J Cancer. 2007;96:373-82.

30. Harada T, Okita K, Shiraishi K, Kusano N, Furuya T, Oga A, et al. Detection of genetic alterations in pancreatic cancers by comparative genomic hybridization coupled with tissue microdissection and degenerate oligonucleotide primed polymerase chain reaction. Oncology. 2002;62:251-8.

31. Nowak NJ, Gaile D, Conroy JM, McQuaid D, Cowell J, Carter R, et al. Genome-wide aberrations in pancreatic adenocarcinoma. Cancer Genet Cytogenet. 2005;161:36-50.

32. Zhang L, Hou Y, Ashktorab H, Gao L, Xu Y, Wu K, et al. The impact of C-MYC gene expression on gastric cancer cell. Mol Cell Biochem. 2010;344:125-35.

33. Dang CV. MYC on the path to cancer. Cell. 2012;149:22-35.

34. Wolf E, Lin CY, Eilers M, Levens DL. Taming of the beast: shaping Mycdependent amplification. Trends Cell Biol. 2015;25:241-8.

35. Felsher DW, Bishop JM. Transient excess of MYC activity can elicit genomic instability and tumorigenesis. Proc Natl Acad Sci USA. 1999;96:3940-4

36. Felsher DW, Bishop JM. Reversible tumorigenesis by MYC in hematopoietic lineages. Mol Cell. 1999:4:199-207.

37. Louis SF, Vermolen BJ, Garini Y, Young IT, Guffei A, Lichtensztejn Z, et al. c-Myc induces chromosomal rearrangements through telomere and chromosome remodeling in the interphase nucleus. Proc Natl Acad Sci USA. 2005;102:9613-8.

38. Yoshioka S, Tsukamoto Y, Hijiya N, Nakada C, Uchida T, Matsuura K, et al. Genomic profiling of oral squamous cell carcinoma by array-based comparative genomic hybridization. PLoS ONE. 2013;8:e56165.

39. Gutenberg A, Gerdes JS, Jung K, Sander B, Gunawan B, Bock HC, et al. High chromosomal instability in brain metastases of colorectal carcinoma. Cancer Genet Cytogenet. 2010;198:47-51.

40. Bao L, Messer K, Schwab R, Harismendy O. Mutational profiling can establish clonal or independent origin in synchronous bilateral breast and other tumors. PLoS ONE. 2015;10:e0142487.

41. Rhim AD, Mirek ET, Aiello NM, Maitra A, Bailey JM, McAllister F, et al. EMT and dissemination precede pancreatic tumor formation. Cell. 2012/01/24 2012;148:349-61.

42. Comen E, Norton L, Massagué J. Clinical implications of cancer selfseeding. Nat Rev Clin Oncol. 2011:8:369-77.

43. Landau DA, Carter SL, Stojanov P, McKenna A, Stevenson K, Lawrence MS, et al. Evolution and impact of subclonal mutations in chronic lymphocytic leukemia. Cell. 2013;152:714-26.

44. Bolli N, Avet-Loiseau H, Wedge DC, Van Loo P, Alexandrov LB, Martincorena l, et al. Heterogeneity of genomic evolution and mutational profiles in multiple myeloma. Nat Commun. 2014;5:2997.

45. Castellarin M, Milne K, Zeng T, Tse K, Mayo M, Zhao Y, et al. Clonal evolution of high-grade serous ovarian carcinoma from primary to recurrent disease. J Pathol. 2013;229:515-24

46. Schuh A, Becq J, Humphray S, Alexa A, Burns A, Clifford R, et al. Monitoring chronic lymphocytic leukemia progression by whole genome sequencing reveals heterogeneous clonal evolution patterns. Blood. 2012;120:4191-6.

47. Johnson BE, Mazor T, Hong C, Barnes M, Aihara K, McLean CY, et al. Mutational analysis reveals the origin and therapy-driven evolution of recurrent glioma. Science. 2014;343:189-93.

48. Klein CA. Selection and adaptation during metastatic cancer progression. Nature. 2013;501:365-72.

49. Anderson K, Lutz C, van Delft FW, Bateman CM, Guo Y, Colman SM, et al. Genetic variegation of clonal architecture and propagating cells in leukaemia. Nature. 2011;469:356-61.

50. Swanton C. Intratumor heterogeneity: evolution through space and time Cancer Res. 2012;72:4875-82.

51. Maley CC, Galipeau PC, Finley JC, Wongsurawat VJ, Li X, Sanchez CA, et al. Genetic clonal diversity predicts progression to esophageal adenocarcinoma. Nat Genet. 2006;38:468-73.

52. Krieg A, Mersch S, Boeck I, Dizdar L, Weihe E, Hilal Z, et al. New model for gastroenteropancreatic large-cell neuroendocrine carcinoma: establishment of two clinically relevant cell lines. PLoS ONE. 2014;9:e88713.

53. Team R. R: A language and environment for statistical computing. Vienna, Austria: R Foundation for Statistical Computing; 2013.

54. Huber W, Carey VJ, Gentleman R, Anders S, Carlson M, Carvalho BS, et al. Orchestrating high-throughput genomic analysis with Bioconductor. Nat Methods. 2015;12:115-21.

55. Ben-Yaacov E, Eldar YC. A fast and flexible method for the segmentation of aCGH data. Bioinformatics. 2008/08/12. 2008;24:1139-45.

56. Hupe P, Stransky N, Thiery J-P, Radvanyi F, Barillot E. Analysis of array CGH data: from signal ratios to gain and loss of DNA regions. Bioinformatics. 2004;20:3413-22.

57. $\mathrm{Mi} \mathrm{H}$, Thomas P. PANTHER pathway: an ontology-based pathway database coupled with data analysis tools. Methods Mol Biol. 2009;563:123-40. 